\title{
Anti-CGRP monoclonal antibodies: a breakthrough in the treatment of migraine
}

\begin{abstract}
To date, the prophylactic treatment of migraine has included only nonspecific drugs of various pharmacological groups: the beta-blockers propranolol and metoprolol, the anticonvulsants topiramate and valproic acid, the antidepressants amitriptyline and venlafaxine, candesartan, and Ona botulinum toxin A. As these drugs were developed for treatment of other diseases, their use was associated with adverse effects: decreased blood pressure, mental retardation, weight increase, nausea, and some others. CGRP is a neuropeptide that was regarded as the main biomarker of migraine as its level in this disease rise. The emergence of humanized monoclonal antibodies has opened up the possibility of blocking the action of CGRP and developing a new class of drugs that includes fremanezumab, erenumab, galcanezumab, and eptinezumab. Anti-CGRP monoclonal antibodies can be prescribed to patients with chronic and episodic migraine. The use of anti-CGRP monoclonal antibodies in clinical studies was associated with a small number of adverse effects, with severe adverse reactions being extremely rare.
\end{abstract}

Keywords: migraine, calcitonin gene related peptide, CGRP, antibody
Volume 9 Issue 5 - 2019

\author{
Julia Azimova, ${ }^{1,2}$ Yaroslav Ashikhmin,' Mikhail \\ Kukushkin, ${ }^{2}$ Aleksandr Amelin, ${ }^{3}$ Eugene \\ Klimov, ${ }^{4,5,6}$ Kirill Skorobogatykh' \\ 'University Headache Clinic LLC, Russian Federation \\ ${ }^{2}$ Institute of General Pathology and Pathophysiology, Russian \\ Federation \\ ${ }^{3}$ The First St. Petersburg State medical University of Acad. I.P. \\ Pavlov Ministry of Health of Russia, Russian Federation \\ ${ }^{4}$ Faculty of Biology, Lomonosov Moscow State University, \\ Russian Federation \\ 5University Diagnostic Laboratory LLC, Russian Federation \\ ${ }^{6}$ Center of Experimental Embryology and Reproductive \\ Biotechnologies, Russian Federation
}

Correspondence: Julia Azimova, University Headache Clinic LLC, I I |467, Moscow, Molodogvardeyskaya Str. 2, BIdg. I, Russian Federation, Russia, Email azimova.j@mail.ru

Received: August 29, 2019 | Published: September 12, 2019
Abbreviations: CGPR, calcitonin gene-related peptide; CNS, central nervous system; RAMP1, receptor activity-modifying protein 1; KATP, ATP-sensitive potassium channel; GI, gastrointestinal

\section{Introduction}

Patients with migraine, a primary headache disorder affecting $15 \%$ of the population; will soon have access to drugs of a new pharmacological class, monoclonal antibodies to calcitonin generelated peptide (CGRP). To date, the prophylactic treatment of migraine has included only nonspecific drugs of various pharmacological groups: the beta-blockers propranolol and metoprolol, the anticonvulsants topiramate and valproic acid, the antidepressants amitriptyline and venlafaxine, candesartan, and Ona botulinum toxin A. As these drugs were developed for treatment of other diseases, their use was associated with adverse effects: decreased blood pressure, mental retardation, weight increase, nausea, and some others. Adverse effects significantly worsen compliance with prophylactic treatment of migraine that should last $6-12$ months. Therefore, migraine is still one of the most maladaptive conditions, which, according to WHO data, is among the top ten diseases with regard to number of years of disability. ${ }^{1}$ An active search for biomarkers of migraine that would enable specific therapy of this disease has been going on for a few decades.

\section{The CGRP system}

The neuropeptide calcitonin gene-related peptide (CGRP) was discovered in $1982^{2}$ and promoted the study of the trigeminovascular system and its role in the development of migraine. CGRP is synthesized in peripheral sensory neurons and some regions of the central nervous system (CNS). There are two forms of CGRP, $\alpha$ and $\beta .^{3} \alpha$-CGRP consists of 37 amino acids, and is synthesized in neurons through tissue-specific mRNA splicing. $\beta$-CGRP is found in the gastrointestinal tract. Transcription begins at the CALCA gene located on chromosome 11. After synthesis, CGRP is transported by vesicles to the axon terminal. ${ }^{4}$ The release of calcitonin gene-related peptide is stimulated by capsaicin. Presynaptic neurons are located on trigeminal neurons, and regulate CGRP release. Activation of 5-HT1B, 5-HT1D, and 5-HT1F serotonin receptors (the target for triptans and ditans, specific agents used to relieve migraine attacks) inhibits the release of CGRP. ${ }^{5}$

After release into the synaptic cleft, calcitonin gene-related peptide is degraded by metalloproteases ${ }^{6}$. Amidation of the carboxyl terminus of the peptide protects it from decomposition, thus prolonging the half-life. This property allows CGRP to reach the receptor and exert its effects some distance from its release site in a process called volume transmission.?

The CGRP receptor is a complex consisting of several proteins each of which is necessary for ligand specificity and function of the receptor itself. The central part of the receptor, G-protein, is the CGRP receptor proper (CALCRL) belonging to the family of secretin receptors. To be able to bind CGRP, the receptor has to form a heterodimer with receptor activity-modifying protein 1 (RAMP 1$).{ }^{8}$ The CGRP binding site is located between the CALCRL and RAMP1 receptors. Therefore, CGRP can affect its receptor only when CALCRL and RAMP1 receptors are co-expressed. ${ }^{3}$ Activation of the receptor results in an increase in intracellular cAMP levels, which triggers phosphorylation of various targets, such as potassium channels (KATP), extracellular signal-regulated kinases, and transcription factors, including CREB. In smooth muscle cells of cerebral blood vessels, CGRP-induced cAMP increase leads to vascular wall relaxation and dilatation of the vessel. ${ }^{9}$ There is also a second CGRP receptor, amylin receptor (AMY1). It consists of RAMP1 and the calcitonin receptor, and is also found in the trigeminal ganglion. The role of this type of receptors in the mechanisms of migraine and in its treatment is unknown. ${ }^{4}$

An important specific feature of CGRP neurotransmission is desensitization of the receptor by agonists. When CGRP binds to the 
CALCRL receptor, the latter is quickly phosphorylated and internalized into endosomes from where it can soon return to the membrane. Chronic exposure to CGRP triggers the process of internalization with capture of the receptor by lysosomes and subsequent destruction. ${ }^{10}$

\section{CGRP and migraine}

The key role of trigeminal sensory fibres in the development of primary headaches was already known at the time when CGRP was discovered. Since CGRP is mainly found in trigeminal neurons, it was thought to play a role in the pathogenesis of migraine. Besides, calcitonin gene-related peptide acts as a potent vasodilator, perfectly fitting into the vascular theory of migraine that was commonly adopted at the time. ${ }^{9}$ In 1990, Goadsby et al. ${ }^{11}$ demonstrated that CGRP is the only neuropeptide whose levels are increased in migraine. Calcitonin gene-related peptide was regarded as a potential diagnostic biomarker of migraine; however, its instability and short half-life precluded development of an informative test. ${ }^{12}$ Intravenous administration of CGRP in patients with migraine triggers a migraine attack, ${ }^{13}$ which allowed modeling of the disease. Finally, CGRP receptor antagonists, gepants, were synthesized and allowed effective relief of migraine attacks. ${ }^{1}$

\section{Anti-CGRP monoclonal antibodies}

Antibody drugs are the mainstream in the development of novel medicines, which is due to: ${ }^{15}$

a. Very high specificity.

b. Optimal pharmacokinetic characteristics that allow drug administration once in 2-5weeks.

c. Significantly better (compared with small molecules) spectrum of adverse effects, including hepatotoxicity, nephro-, cardio-, and neurotoxicity (except where due to direct effects on the target).

d. Greater opportunities for protection of intellectual property, as antibodies are difficult to "copy".

\section{Efficacy of anti-CGRP monoclonal antibodies}

The first anti-CGRP monoclonal antibodies were synthesized after the discovery of the peptide in 1982, but these were used for diagnostic purposes in liquid chromatography and immunohistochemistry to study CGRP in tissues. ${ }^{2}$ The emergence of humanized monoclonal antibodies has opened up the possibility of blocking the action of CGRP and developing a new class of drugs that includes fremanezumab, galcanezumab, and eptinezumab. ${ }^{4}$ The development of anti-CGRP receptor monoclonal antibodies also provided a new approach to blocking the receptor. Erenumab is a human monoclonal antibody to the extracellular domains of CALCRL and RAMP1. ${ }^{16}$ Fremanezumab, galcanezumab, and erenumab are available for subcutaneous injection, and eptinezumab for intravenous infusion. The maximum serum concentration (Cmax) of the subcutaneous dosage forms is 4-13days, while the Cmax of the intravenous eptinezumab is achieved on the day of administration. The short time to maximum concentration results in quick onset of action of the drugs. The elimination half-life is in the range of 25-32days, so the drugs can be given once a month. ${ }^{17-19}$ Monoclonal antibodies are eliminated by the reticuloendothelial system.

All drugs based on monoclonal antibodies to CGRP or to its receptor have shown their efficacy in both episodic and chronic migraine, decreasing the frequency of both days of headache and days of migraine. In the STRIVE trial, 995 patients with episodic migraine were administered erenumab $70 \mathrm{mg}$, erenumab $140 \mathrm{mg}$, or placebo once monthly for 6 months. A $50 \%$ or greater reduction in migraine days at treatment months $4-6$ was observed in $43.3 \%$ of patients treated with erenumab $70 \mathrm{mg}$, in $50 \%$ of subjects in the erenumab $140 \mathrm{mg}$ arm, and in $26.6 \%$ of placebo-treated patients $(\mathrm{p}<0.001$ for comparisons between each of the dose levels and placebo). ${ }^{11}$ The LIBERTY trial evaluated the efficacy of erenumab in patients with episodic migraine after failure of one or two drugs for the prevention of migraine. In this patient population, a $50 \%$ or greater reduction in migraine days was observed in $30.3 \%$ of patients treated with erenumab $140 \mathrm{mg}$ and in $13.7 \%$ of placebo-treated subjects $(\mathrm{p}=0.002) .{ }^{11}$

Eptinezumab was investigated in two studies, PROMISE 1 (episodic migraine) and PROMISE 2 (chronic migraine). In the PROMISE 1 protocol, patients received eptinezumab 30mg, eptinezumab $100 \mathrm{mg}$, eptinezumab $300 \mathrm{mg}$, or placebo. A $50 \%$ or greater reduction in migraine days at treatment week 12 was observed in $50.2 \%$ of patients administered eptinezumab $30 \mathrm{mg} \quad(\mathrm{p}=0.006)$, $49.8 \%$ of subjects in the eptinezumab $100 \mathrm{mg}$ group $(\mathrm{p}=0.009)$, and $56.3 \%$ of patients receiving eptinezumab $300 \mathrm{mg} .^{20}$

HALO was the pivotal efficacy and safety study of fremanezumab in episodic and chronic migraine. Subjects with episodic migraine were administered fremanezumab $675 \mathrm{mg}$ quarterly or fremanezumab $225 \mathrm{mg}$ monthly or placebo. A $50 \%$ or greater reduction in migraine days was observed in $44.4 \%$ of patients treated with quarterly fremanezumab, in $47.7 \%$ of patients given monthly fremanezumab, and in $27.9 \%$ of placebo-treated patients $(\mathrm{p}<0.001$ for comparisons between each of the dose levels and placebo). ${ }^{21}$ In the same study, patients with chronic migraine were given fremanezumab $675 \mathrm{mg}$ quarterly and $225 \mathrm{mg}$ at weeks 4 and 8, fremanezumab $675 \mathrm{mg}$ quarterly and placebo at weeks 4 and 8 , or placebo. A $50 \%$ or greater reduction in migraine days was observed in $38 \%$ of patients treated with quarterly fremanezumab, in $41 \%$ of subjects given monthly fremanezumab, and in $18 \%$ of placebo-treated patients ( $p<0.001$ for comparisons between each of the dose levels and placebo). ${ }^{22}$

The efficacy of galcanezumab $120 \mathrm{mg}$ and $240 \mathrm{mg}$ versus placebo in patients with episodic or chronic migraine was assessed in the EVOLVE-1 and REGAIN studies. In episodic migraine, a 50\% or greater reduction in migraine days at 6 months was observed in $62.3 \%$ of patients treated with galcanezumab $120 \mathrm{mg}$, in $62.3 \%$ of subjects given galcanezumab $240 \mathrm{mg}$, and in $42.5 \%$ of placebo-treated patients $(p<0.001$ for comparisons between each of the dose levels and placebo). ${ }^{23}$

New efficacy endpoints, proportions of patients with $75 \%$ and $100 \%$ reductions in migraine days, were used for the first time in antiCGRP studies.

\section{Practical aspects of the use of anti-CGRP monoclonal antibodies}

Anti-CGRP monoclonal antibodies can be prescribed to patients with chronic and episodic migraine. These drugs are mostly indicated for patients with pre-chronic migraine (8-14days of migraine a month), as well as for patients with chronic migraine or episodic migraine with an average frequency of attacks (4-7days of migraine a month). ${ }^{24}$ These agents should be considered for patients who cannot be administered standard therapy due to comorbidities, adverse effects 
and low compliance. ${ }^{25}$ Monoclonal antibodies can prove effective after failure of one or two standard preventive migraine drugs. ${ }^{26}$

The switch from standard therapy to treatment with monoclonal antibodies has some specific aspects. It is recommended to discontinue standard therapy before initiating antibodies in patients with episodic migraine. In chronic migraine or episodic migraine in a patient with a history of chronic migraine, anti-CGRP antibodies should be administered without discontinuing standard therapy. After that, once an effect has been achieved, standard therapy may be discontinued on an individual basis. The recommended duration of monoclonal antibody therapy is 6-12months. In the presence of concurrent medication-overuse headache, monoclonal antibodies may be started either before or after detoxification. The main contraindications to the use of monoclonal antibodies are pregnancy and lactation; alcohol dependence and other types of dependences; relevant cardiac and cerebrovascular disorders; severe psychiatric disorders. ${ }^{25}$

\section{Safety issues associated with the use of anti- CGRP monoclonal antibodies}

\section{Safety of the antibody platform of anti-CGRP drugs}

Hazards associated with the use of monoclonal antibodies may be due to specific effects on the target (see below) or abnormal response of the immune system. The latter may vary in severity from mild reactions of chills and fever to a potentially fatal cytokine storm. Other disadvantages of antibody drugs include their potential immunogenicity (formation of anti-drug antibodies potentially leading to decreasing efficacy in long-term use) and high cost of manufacturing. Both immunogenicity and the risk of abnormal immune response depend on the type of the antibody. Apparently, the risk is highest with mouse antibodies and lowest with human ones (which are usually more expensive to manufacture). Chimeric/ humanized monoclonal antibodies are between the two extremes in this regard. ${ }^{27}$

The IgG subclass that the antibody belongs to is important as well: IgG1 is significantly more likely to activate the complement system (which is undesirable in the treatment of migraine) than IgG4 and IgG2 ${ }^{28}$ On the other hand, IgG2 has the lowest potential antibodydependent cell-mediated cytotoxicity, so this antibody class is most suitable for the manufacture of drugs for the treatment of migraine.

Based on the properties of the antibodies in question, one can assume that the humanized antibody fremanezumab and the human antibody erenumab, both of the IgG2 subclass, can have the best safety profile and the lowest immunogenicity. ${ }^{29}$ This was confirmed by results of Phase II studies in which the frequency of immunogenicity reactions was found to be $1 \%$ for fremanezumab, ${ }^{29} 6 \%$ for erenumab, ${ }^{27} 12 \%$ for eptinezumab ${ }^{29}$ and up to $30 \%$ for galcanezumab. The study's authors underline that the development of autoantibodies to galcanezumab did not result in any changes in blood concentrations of the drug or CGRP in the Phase II studies. ${ }^{30}$

Drugs with lower risk of immunogenicity may be potentially preferable for patients with chronic migraine who may require longer use of anti-CGRP monoclonal antibodies.

It is essential to note that anti-CGRP monoclonal antibodies exhibit no impact on the central nervous system, as their high molecular weight makes them unable to cross the blood-brain barrier. ${ }^{4}$

\section{General safety considerations associated with the use of anti-CGRP monoclonal antibodies}

The spectrum of adverse effects of anti-CGRP monoclonal antibodies is presented in Table $1 .{ }^{31}$

Table I The most frequent adverse effects and adverse reactions reported during Phase II and III clinical trials (CTs) of anti-CGRP MAs

\begin{tabular}{|c|c|c|c|c|}
\hline Drug & Fremanezumab & Erenumab & Galcanezumab & Eptinezumab \\
\hline $\begin{array}{l}\text { Common adverse } \\
\text { reactions (incidence } \\
I-10 \% \text { ) }\end{array}$ & $\begin{array}{l}\text { Injection site reactions (up } \\
\text { to } 45 \% \text { ) }\end{array}$ & $\begin{array}{l}\text { Injection site } \\
\text { reactions (3-6\%) } \\
\text { Constipation } \\
\text { Muscle cramps } \\
\text { Weakness }\end{array}$ & $\begin{array}{l}\text { Injection site reactions } \\
(18 \%)\end{array}$ & $\begin{array}{l}\text { Equal to or less than } \\
\text { placebo }\end{array}$ \\
\hline
\end{tabular}

Hypersensitivity reactions, such as rashes, angioneurotic oedema, and anaphylaxis, were also observed with all anti-CGRP MAs; their occurrence did not exceed that with other antibodies. Other adverse reactions identified during phase III CTs included nasopharyngitis and upper respiratory tract infections, urinary tract infection, dizziness, nausea, and arthralgia. The frequency of these adverse effects was comparable to the placebo arm. As eptinezumab was administered intravenously, no complications associated with subcutaneous administration were detected. Reactions at the injection site were evaluated in the studies in different ways: either all reactions were evaluated as one adverse event, or pain at the injection site, redness, infiltration, etc. were reported separately. Regardless of the method of evaluation, there was no difference with placebo.

The use of anti-CGRP monoclonal antibodies in clinical studies was generally associated with a small number of adverse effects, with severe adverse reactions being extremely rare. Below we will discuss safety issues associated with the specific inhibition of the CGRP system resulting from binding of therapeutic antibodies to the ligand or CGRP receptor.

\section{Gastrointestinal safety of anti-CGRP monoclonal antibodies}

The gastrointestinal (GI) tract has massive CGRPergic innervation. Its functions include regulation of gastrointestinal motility and protection of the mucous membrane from deleterious factors. ${ }^{32}$ In animal experiments, exposure to anti-CGRP monoclonal antibodies resulted in pronounced damage to the gastrointestinal mucous lining. ${ }^{33}$ Analysis of available clinical study results does not reveal any increase in the incidence of gastrointestinal adverse effects during treatment with anti-CGRP monoclonal antibodies, as compared with placebo. In particular, in the Phase III study of erenumab, ARICE, constipation was observed in $1.4 \%$ of erenumab-treated patients and in $2.1 \%$ subjects of the placebo group. ${ }^{34}$ According to Medscape data, constipation can occur in $3 \%$ of patients treated with erenumab medscape.com. 


\section{Cardiovascular safety of anti-CGRP monoclonal antibodies}

CGRP plays a considerable role in the regulation of cardiovascular function, both in the central and peripheral nervous system and directly at the level of the heart and vessels. ${ }^{32}$ CGRP receptors are expressed in peripheral arteries and the heart; besides, they are innervated by CGRPergic nerve fibres.

To date, experimental studies have yielded the following aspects of the effects of CGRP and its agonists on the cardiovascular system:

1. CGRP is a potent vasodilator, and exerts positive inotropic and chronotropic effects; $;^{35}$

2. CGRP gene knockout mice used in various hypertension models demonstrated significantly elevated blood pressure levels and more profound involvement of the target organs of hypertension compared with control animals, ${ }^{36}$

3. Use of CGRP in a rat acute cerebral artery occlusion model was associated with less severe cerebral oedema; ${ }^{35}$

4. Use of a slow-release system to deliver CGRP into the cerebrospinal fluid in a monkey subarachnoid haemorrhage model was associated with relieved vascular spasm. ${ }^{38}$ Similarly, administration of CGRP in patients with subarachnoid haemorrhage was associated with decreased severity of vascular spasm; ${ }^{39}$

5. In biological models of myocardial infarction, CGRP decreased the volume of ischaemic myocardium and prevented the development of life-threatening arrhythmias; ${ }^{40}$

6. Recently developed CGRP analogues demonstrated an antihypertensive effect, prevented pathological myocardial remodeling, and stimulated angiogenesis in biological models of hypertension and heart failure; ${ }^{41}$

7. Use of CGRP in patients with congestive heart failure resulted in improved pumping capacity of the heart. ${ }^{42}$

These experiments, as well as a number of others that are not mentioned in this paper, allow a conclusion that CGRP has cardioprotective and vasoprotective properties. Accordingly, the initially cautious use of anti-CGRP monoclonal antibodies was due to the risk of vasoconstriction, which in turn can elevate blood pressure and induce cerebral and myocardial ischaemia.

As a result, a programme was started to study the effects of CGRP antagonists on the cardiovascular system. In the overwhelming majority of experiments in biological models, use of CGRP antagonists did not affect haemodynamic parameters and had no effect on the severity of ischaemia in arterial occlusion. ${ }^{43}$ A study in a pithed rat model found that the CGRP antagonist olcegepant enhanced sympathetic adrenergic effects, which resulted in elevated blood pressure. ${ }^{44}$ Administration of CGRP antagonists in healthy volunteers did not increase blood pressure or have any other negative consequences either. ${ }^{45}$

The most important clinical trial data were obtained for antiCGRP monoclonal antibodies in Phase II and III studies that included office blood pressure monitoring and ECG recording. None of the studies, including a long-term follow-up (for more than 1.5years), has yet been able to demonstrate any negative effect of anti-CGRP monoclonal antibodies on the cardiovascular system. It should be noted that the clinical studies of anti-CGRP monoclonal antibodies mainly recruited female patients at very low risk of cardiovascular events. To study the effects of these drugs in patients at high risk, erenumab was investigated in a separate double-blind, placebocontrolled, randomized study that enrolled 90 subjects with stable angina, including patients after acute myocardial infarction. ${ }^{46} \mathrm{~A}$ stress test revealed that erenumab $140 \mathrm{mg}$ i.v. had no effect on physical exercise tolerance or development of myocardial ischaemia. It was concluded that CGRP receptor blockade is safe for the cardiovascular system. The authors underlined that erenumab blocks only the canonical CGRP receptor without exerting any effect on the binding of CGRP to the amylin 1 receptor for which the former shows high affinity. These data are consistent with results that were obtained in a study of telcagepant in patients with stable angina pectoris. The use of telcagepant did not result in myocardial ischaemia, as revealed by a stress test, either ${ }^{47}$ Therefore, the available data imply a rather good medium-term cardiovascular safety profile of anti-CGRP monoclonal antibodies in a typical population of migraine patients.

\section{Further steps in the study of the cardiovascular safety of anti-CGRP monoclonal antibodies}

Notwithstanding the promising results of a single study of erenumab in patients with stable ischaemic heart disease, the question of the safety of anti-CGRP monoclonal antibodies in patients at high risk of cardiovascular complications, particularly in the long term, is still open to debate. Currently these agents are contraindicated in patients with serious cardiovascular diseases. It is advisable to include 24-hour blood pressure monitoring in the protocols of new largescale studies of CGPR inhibitors. In small-scale studies that enroll patients with heart disease, cardiac MRI with myocardial perfusion assessment and analysis of biomarkers (such as ultra-high sensitivity troponin and NT-pro-BNP) are the best tools to clearly demonstrate the absence of cardiac toxicity. The role of endothelial dysfunction in the pathogenesis of migraine and the influence of anti-CGRP monoclonal antibodies are not clearly understood yet, which warrants endothelial function studies in migraine patients treated with these drugs.

\section{Acknowledgments}

This research received no specific grant from any funding agency in the public, commercial, or not-for-profit sectors.

\section{Conflicts of interest}

The author declares no conflict of interest.

\section{Funding}

None.

\section{References}

1. GBD 2016 Disease and Injury Incidence and Prevalence Collaborators Global, regional, and national incidence, prevalence, and years lived with disability for 328 diseases and injuries for 195 countries, 1990-2016: a systematic analysis for the global burden of disease study 2016. Lancet. 2017;390(10100):1211-1259.

2. Amara SG, Jonas V, Rosenfeld MG, et al. Alternative RNA processing in calcitonin gene expression generates mRNAs encoding different polypeptide products. Nature. 1982;298(5871):240-244.

3. Russell FA, King R, Smillie SJ, et al. Calcitonin gene- related peptide: physiology and pathophysiology. Physiol Rev. 2014;94(4):1099-1142. 
4. Edvinsson L, Haanes KA, Warfvinge K, et al. CGRP as the target of new migraine therapies - successful translation from bench to clinic. Nat Rev Neurol. 2018;14(6):338-350.

5. Raffaell B, Israel H, Neeb L, et al. The safety and efficacy of the 5-HT 1 $\mathrm{F}$ receptor agonist lasmiditan in the acute treatment of migraine. Expert Opin. Pharmacother. 2017;18(13):1409-1415.

6. Kim YG, Lone AM, Saghatelian A. Analysis of the proteolysis of bioactive peptides using a peptidomics approach. Nat Protoc. 2013;8(9):1730-1742.

7. Russo AF. Overview of neuropeptides: awakening the senses? Headache. 2017;57 Suppl. 2:37-46.

8. McLatchie LM, Fraser NJ, Main MJ, et al. RAMPs regulate the transport and ligand specificity of the calcitonin- receptor-like receptor. Nature. 1998;393(6683):333-339.

9. Edvinsson L, Fredholm BB, Hamel E, et al. Perivascular peptides relax cerebral arteries concomitant with stimulation of cyclic adenosine monophosphate accumulation or release of an endothelium- derived relaxing factor in the cat. Neurosci Lett. 1985;58(2):213-217.

10. Padilla BE, Cottrell GS, Roosterman D, et al. Endothelin- converting enzyme-1 regulates endosomal sorting of calcitonin receptor-like receptor and beta-arrestins. J Cell Biol. 2007;179(5):981-997.

11. Goadsby PJ, Reuter J, Hallstrom Y, et al. A controlled trial of erenumab for episodic migraine. N Engl J Med. 2017;377(22):2123-2132.

12. Edvinsson L, Ekman R, Goadsby PJ. Measurement of vasoactive neuropeptides in biological materials: problems and pitfalls from 30 years of experience and novel future approaches. Cephalalgia. 2010;30(6):761-766.

13. Hansen JM, Hauge AW, Olesen J, et al. Calcitonin gene- related peptide triggers migraine-like attacks in patients with migraine with aura. Cephalalgia. 2010;30(10):1179-1186.

14. Bell IM. Calcitonin gene- related peptide receptor antagonists: new therapeutic agents for migraine. J Med Chem. 2014;57(19):7838-7858.

15. Ritter L, Flower R, Henderson G, et al. Rang \& Dale's Pharmacology. 8th edn. UK: Churchill Livingstone; 2015. p. 776.

16. Shi L, Lehto SG, Zhu DX, et al. Pharmacologic characterization of AMG 334, a potent and selective human monoclonal antibody against the calcitonin gene- related peptide receptor. J Pharmacol Exp Ther. 2016;356(1):223-231.

17. Monteith D, Collins EC, Vandermeulen C, et al. Safety, tolerability, pharmacokinetics, and pharmacodynamics of the CGRP binding monoclonal antibody LY2951742 (Galcanezumab) in healthy volunteers. Front Pharmacol. 2017;8:740.

18. Alder-Announces-Eptinezumab-Significantly-Reduces-MigraineRisk-Meets-Primary-and-All-Key-Secondary-Endpoints-in-PivotalPROMISE-2-Phase-3-Trial-for-Chronic-Migraine-Prevention. USA: Alder BioPharmaceuticals; 2018.

19. Reuter U, Goadsby PJ, Lanteri-Minet M, et al. Efficacy and tolerability of erenumab in patients with episodic migraine in whom two-to-four previous preventive treatments were unsuccessful: a randomised, double-blind, placebo-controlled, phase 3b study. Lancet. 2018;392(10161):2280-2287.

20. Saper J, Lipton RB, Kudrow D, et al. A Phase 3, randomized, doubleblind, placebo-controlled study to evaluate the efficacy and safety of eptinezumab in frequent episodic migraine prevention: Primary results of the PROMISE 1 (prevention of migraine via intravenous eptinezumab safety and efficacy 1) trial. Cephalalgia. 2017;37:337.

21. Dodick DW, Silberstein SD, Bigal ME, et al. Effect of fremanezumab compared with placebo on prevention of episodic migraine: A randomized clinical trial. JAMA. 2018;319(19):1999-2008.
22. Silberstein SD, Dodick DW, Bigal ME, et al. Fremanezumab for the preventive treatment of chronic migraine. $N$ Engl $\mathrm{J} \mathrm{Med}$. 2017;377(22):2113-2122.

23. Skljarevski V, Stauffer VL, Zhang Q, et al. Phase 3 studies (EVOLVE-1 \& EVOLVE-2) of galcanezumab in episodic migraine: results of 6 month treatment phase. Cephalalgia. 2017;37:339-340.

24. Martelletti P. The application of CGRP(r) Monoclonal antibodies in migraine spectrum: needs and priorities. BioDrugs. 2017;31(6):483-485.

25. Sacco S, Bendtsen L, Ashina M, et al. European headache federation guideline on the use of monoclonal antibodies acting on the calcitonin gene related peptide or its receptor for migraine prevention. J Headache Pain. 2019;20(1):6.

26. Ashina M, Tepper S, Brandes JL, et al. Efficacy of erenumab (a fully human Mab targeting the CGRP receptor) in chronic migraine patients with prior treatment failure: a subgroup analysis of the phase 2, randomized, double-blind, placebo-controlled study. Cephalalgia. 2017; 38(10):1611-1621.

27. Vargas B, Starling A, Silberstein S, et al. Erenumab immunogenicity: a pooled analysis of phase 2 and phase 3 migraine prevention clinical trials. Neurology. 2018;90(Suppl 15):4.098.

28. Presta LG. Engineering of therapeutic antibodies to minimize immunogenicity and optimize function. Adv Drug Deliv Rev. 2006;58(56):640-656.

29. Taylor F. CGRP, amylin, immunology, and headache medicine. Headache. 2019;59(1):131-150.

30. Oakes T, Skljarevski V, Zhang Q, et al. Safety of galcanezumab in patients with episodic migraine: A andomized placebo-controlled doseranging Phase 2b study. Cephalalgia. 2018;38(6):1015-1025.

31. Mavridis T, Koniari C, Fakas N, et al. Anti-calcitonin gene-related peptide monoclonal antibodies: adverse effects. what do we really know? A literature review. EMJ Innov. 2019;3(1):64-72.

32. Deen M, Correnti E, Kamm K, et al. Blocking CGRP in migraine patients - a review of pros and cons. J Headache Pain. 2017;18(1):96.

33. Peskar BM, Wong HC, Walsh JH, et al. A monoclonal antibody to calcitonin gene-related peptide abolishes capsaicin-induced gastroprotection. Eur J Pharmacol. 1993;250(1):201-203.

34. Dodick D, Ashina M, Brandes J, et al. ARISE: a Phase 3 randomized trial of erenumab for episodic migraine. Cephalalgia. 2018; 38(6):10261037.

35. Rubio-Beltran E, van den Brink AM. Understanding CGRP and cardiovascular risk. Handb Exp Pharmacol. 2019;255:131-140.

36. Jianping L, Kevin AC, Donald JD, et al. Renal protective effects of $\alpha-$ calcitonin generelated peptide in deoxycorticosterone-salt hypertension. Am J Physiol Renal Physiol. 2013;304(7):F1000-1008.

37. Liu Z, Liu Q, Cai H, et al. Calcitonin gene-related peptide prevents blood-brain injury and brain edema induced by focal cerebral ischemia reperfusion. Regul Pept. 2011;171(1-3):19-25.

38. Inoue T, Shimizu H, Kaminuma T, et al. Prevention of cerebral vasospasm by calcitonin gene-related peptide slow-release tablet after subarachnoid hemorrhage in monkeys. Neurosurgery. 1996;39(5):984-990.

39. Juul R, Aakhus S, Björnstad K, et al. Calcitonin gene-related peptide (human alpha-CGRP) counteracts vasoconstriction in human subarachnoid haemorrhage. Neurosci Lett. 1994.170(1):67-70.

40. Xiao ZS, Li YJ, Deng HW. Ischemic preconditioning mediated by calcitonin gene-related peptide in isolated rat hearts. Zhongguo Yao Li Хие Bao. 1996;17(5):445-448.

41. Aubdool AA, Thakore P, Argunhan F, et al. A novel alphacalcitonin 
gene-related peptide analogue protects against end-organ damage in experimental hypertension, cardiac hypertrophy, and heart failure. Circulation. 2017;136(4):367-383.

42. Gennari C, Nami R, Agnusdei D, et al. Improved cardiac performance with human calcitonin gene related peptide in patients with congestive heart failure. Cardiovasc Res. 1990;24(3):239-241.

43. Favoni V, Giani L, Al-Hassany L, et al. CGRP and migraine from a cardiovascular point of view: what do we expect from blocking CGRP? J Headache Pain. 2019;20(1):27.

44. Avilés-Rosas VH, Rivera-Mancilla E, Marichal-Cancino BA, et al. Olcegepant blocks neurogenic and non-neurogenic CGRPergic vasodepressor responses and facilitates noradrenergic vasopressor responses in pithed rats. Br J Pharmacol. 2017;174(13):2001-2014.
45. Petersen KA, Birk S, Lassen LH, et al. The CGRP-antagonist, BIBN4096BS does not affect cerebral or systemic haemodynamics in healthy volunteers. Cephalalgia. 2005;25(2):139-147.

46. Depre C, Antalik L, Starling A, et al. A randomized, double-blind, placebo-controlled study to evaluate the effect of Erenumab on exercise time during a treadmill test in patients with stable angina. Headache. 2018;58(5):715-723.

47. Cui X, Ye J, Lin H, et al. Efficacy, safety, and tolerability of telcagepant in the treatment of acute migraine: a meta-analysis. Pain Pract. 2015;15(2):124-131. 